\title{
Technical Feasibility and Application of Mechanical Thrombectomy with the Solitaire FR Revascularization Device in Acute Basilar Artery Occlusion
}

\author{
P. Mordasini, C. Brekenfeld, J.V. Byrne, U. Fischer, M. Arnold, M.R. Heldner, R. Lüdi, H.P. Mattle, G. Schroth, and J. Gralla
}

\begin{abstract}
BACKGROUND AND PURPOSE: Acute BAO is a devastating neurological condition associated with a poor clinical outcome and a high mortality rate. Recanalization has been identified as a major prognostic factor for good outcome in BAO. Mechanical thrombectomy using retrievable stents is an emerging treatment option for acute stroke. First clinical trials using stent retrievers have shown promising high recanalization rates. However, these studies mainly included large artery occlusions in the anterior circulation with only a few or single cases of BAO. Therefore, the purpose of this study was to evaluate technical feasibility, safety, and efficacy of mechanical thrombectomy using retrievable stent in the treatment of acute $\mathrm{BAO}$.
\end{abstract}

MATERIALS AND METHODS: Fourteen consecutive patients with BAO undergoing endovascular therapy using retrievable stents (Solitaire FR Revascularization Device) were included. Additional multimodal treatment approaches included thromboaspiration, intravenous and/or intra-arterial thrombolysis, and PTA/ permanent stent placement. Recanalization rates after multimodal therapy and stent retrieval were determined. Clinical outcome and mortality were assessed 3 months after treatment.

RESULTS: Median patient age was 64.5 years (range 55-85). Median NIHSS score at presentation was 21 (range 5-36). Overall, successful recanalization ( $\mathrm{TICl} 3$ or $2 \mathrm{~b}$ ) was achieved in all patients ( $\mathrm{TICI} 3$ in $78.6 \%$, 11/14). In 4 patients (28.6\%), insufficient recanalization after stent retrieval was due to an underlying atherosclerotic stenosis. Additional deployment of a permanent intracranial stent was performed in 3 patients (21.4\%) and PTA alone in 1 patient (7.1\%), resulting in final $\mathrm{TICl} 3$ in 1 patient and $\mathrm{TICl} 2 \mathrm{~b}$ in 3 patients. Stent retrieval alone was performed in 4 patients (28.6\%). Average number of device passes was 1.3 (range 1-3). Median procedure time to maximal recanalization was 47 minutes (range 10-252). No device-related complications or thromboembolic occlusion of a previously unaffected artery occurred. There was no symptomatic intracranial hemorrhage. At 3 months, good functional outcome (mRS 0-2) was observed in $28.6 \%$ (4/14); overall mortality was $35.7 \%$ (5/14).

CONCLUSIONS: A multimodal endovascular approach using retrievable stents in BAO has high recanalization rates, with very low complication rates. Underlying atherothrombotic stenotic lesions of the basilar artery may still necessitate additional permanent stent placement to achieve complete recanalization.

ABBREVIATIONS: $B A O=$ basilar artery occlusion; $\mathrm{mRS}=$ modified Rankin Scale; PROACT = Prolyse in Acute Cerebral Thromboembolism study; PTA = percutaneous transluminal angioplasty; TICl = Thrombolysis in Cerebral Infarction; TIMI = Thrombolysis in Myocardial Infarction; TOAST = Trial of Org 10172 in Acute Stroke Treatment

A cute BAO is a devastating condition associated with poor clinical outcome and mortality rates approaching $90 \%$ in patients without treatment. ${ }^{1-4}$ Recanalization is the most important prognostic factor for good functional outcome in BAO. ${ }^{5-8}$ Recanalization rates with intravenous or intra-arterial thrombolysis are

Received January 10, 2012; accepted after revision March 9

From the Institute of Diagnostic and Interventional Neuroradiology (P.M., C.B., G.S., J.G.), Inselspital, and Department of Neurology (U.F., M.A., M.R.H., R.L., H.P.M.), University of Bern, Switzerland; and Oxford Neurovascular and Neuroradiology Research Unit (J.V.B.), Nuffield Department of Surgery, The John Radcliffe Hospital, University of Oxford, United Kingdom.

Please address correspondence to Pasquale Mordasini, MD, Department of Diagnostic and Interventional Neuroradiology, Inselspital, University of Bern,

Freiburgstr 4, CH-3010 Bern, Switzerland; e-mail: pasquale.mordasini@insel.ch

http://dx.doi.org/10.3174/ajnr.A3168 approximately $53 \%$ and $65 \%$, respectively, and chances of good outcome without recanalization are only around $2 \% .{ }^{9}$ Mechanical thrombectomy using self-expanding retrievable stents is an emerging treatment option for acute stroke. It combines the fast and efficient flow restoration effect of an intracranial stent and the capability of definitive thrombus removal of a mechanical thrombectomy device. The Solitaire FR Revascularization Device (ev3, Irvine, California) is the first dedicated retrievable stent device for acute stroke treatment. At our institution, in January 2010, stent retrievers were added to our multimodal endovascular stroke treatment protocol. Recent data of our own have demonstrated that adding retrievable stents to a multimodal approach in acute ischemic stroke treatment reduces the time to recanalization and further increases the recanalization rate to over $90 \% .^{10}$ 
However, most studies concerning the efficacy of stent retrievers have been performed in large vessel occlusions of the anterior circulation, including only a few or single cases of BAO. ${ }^{10-17}$ Furthermore, in the posterior circulation, proximal balloon occlusion for flow reversal during retrieval is not applicable. Therefore, the risk of distal thromboembolic events is potentially increased. Hence, clinical data and detailed analyses focusing on the impact of mechanical thrombectomy using stent retrievers in BAO are still sparse. ${ }^{14,15}$

The purpose of this study was to evaluate the feasibility, safety, and efficacy of retrievable stents, with special focus on their application in the treatment of acute BAO.

\section{MATERIALS AND METHODS}

This study is based on the Bernese stroke registry. Since 1992, data for all patients with stroke treated by endovascular means are prospectively recorded in our stroke data base. From this prospectively collected cohort, we retrospectively analyzed all patients with acute ischemic stroke with BAO undergoing endovascular stroke treatment between January 2010 and August 2011. All patients were evaluated on admission by a stroke neurologist and assessed with the NIHSS score. Inclusion criteria for endovascular stroke treatment were as follows: 1) diagnosis of BAO was established; 2) baseline NIHSS score was $\geq 4$ or hemianopia was present; 3) hemorrhage on cranial CT or MR imaging was excluded; 4) $\mathrm{BAO}$, as observed on DSA, correlated with the neurologic deficit; 5) symptom duration was not $>24$ hours; and 6) no individual clinical or premorbid conditions or laboratory findings advised against recanalization.

Multimodal treatment consisted of a combination of 1 ) thromboaspiration, 2) intravenous and/or intra-arterial thrombolysis, 3) mechanical thrombectomy, and 4) PTA/stent placement. Intravenous thrombolysis with rtPA (full dose $0.9 \mathrm{mg} / \mathrm{kg}$ ) was administered as bridging therapy in patients referred within 4.5 hours from symptom onset. A loading dose of $10 \%$ was given in these patients. Intra-arterial urokinase (Urokinase Medac; Medac, Wedel, Germany) was used up to a dose of 1,000,000 IU within the first 6 hours after symptom onset.

\section{Endovascular Treatment}

An $8 \mathrm{~F}$ sheath was placed in the femoral artery, and a 4-vessel diagnostic DSA was performed to assess vessel occlusion and collateral flow to the affected vessel territory. After confirmation of vessel occlusion, the largest possible guide catheter to allow safe catherization, a 6-8F guide catheter (Guider Softip; Boston Scientific, Natick, Massachusetts), was introduced into the dominant or most accessible vertebral artery.

For thromboaspiration, the guide catheter was advanced distally in the vertebral artery. Then an aspiration catheter $(5.1 \mathrm{~F}$ Vasco35+ASPI, Balt, Montmorency, France, or 5.2F DAC, Concentric Medical, Mountain View, California) was advanced to the occlusion site. Aspiration was performed by 2 lockable $60-\mathrm{mL}$ syringes connected to the guide and aspiration catheters. The aspiration catheter was removed during continuous aspiration of both catheters.

Intra-arterial thrombolysis was performed via a microcatheter (2.8F Renegade Hi-Flo, Boston Scientific; or 2.3F Prowler Select
Plus, Cordis, Miami Lakes, Florida) for 30-60 minutes by placing the catheter tip into the occluding thrombus. Follow-up angiograms were performed every 20-30 minutes to assess patency.

Mechanical thrombectomy was performed using the Solitaire FR Revascularization Device (ev3). All retrievable stents used measured $4 \mathrm{~mm}$ in diameter and $20 \mathrm{~mm}$ in length. First, the thrombus was passed with the microwire (SilverSpeed 14; ev3) and the microcatheter (2.3F Prowler Select Plus) and a distal angiogram was performed to confirm proper localization in the target cerebral artery. The Solitaire FR was placed through the microcatheter and deployed by retracting the microcatheter through the occlusion site under fluoroscopic control. Once fully deployed, a control angiogram was obtained to assess immediate recanalization effect. According to the results of previous animal and clinical studies, ${ }^{10,18}$ the device was left in place for 5 minutes to allow the clot to become embedded in the stent before the microcatheter was then advanced to partially resheath the proximal connection site of the stent to the pusher wire. The Solitaire FR and the microcatheter were retrieved simultaneously during aspiration with a $60-\mathrm{mL}$ syringe at the guide catheter. If this failed or recanalization was insufficient, the device was cleaned and reinserted. A maximum of 3 retrieval attempts was allowed per device.

In cases where no sufficient recanalization was achieved, despite various recanalization attempts, placement of an intracranial stent (Wingspan; Boston Scientfic) and/or PTA was applied to reduce the risk of delayed reocclusion.

Control angiograms were performed after each revascularization attempt to assess the recanalization results and thromboembolic events in a previously unaffected vessel territory. The extent of recanalization was classified according to the TICI grading scale. ${ }^{19}$ TICI grades 3 and $2 \mathrm{~b}$ were rated as sufficient recanalization, whereas TICI grades $2 \mathrm{a}$ to 0 were rated as insufficient recanalization.

All endovascular procedures were performed under general anesthesia. Postprocedure patients were observed for at least 24 hours in an intensive care unit, and CT or MR imaging was performed if clinical worsening occurred to rule out intracranial hemorrhage. The NIHSS score was assessed at day 1 by a stroke neurologist. Clinical outcome was assessed at 3 months by a stroke neurologist according to the mRS. ${ }^{20}$ An mRS score of $0-2$ was defined as good and an mRS score of 3-6 was defined as poor functional outcome. Stroke etiology was classified according to TOAST criteria. ${ }^{21}$ The following time points were recorded for analysis: onset of symptoms, first angiogram, and achievement of final recanalization result. Time intervals from symptom onset to the first angiogram and procedure time to final recanalization (time to recanalization) were calculated.

\section{RESULTS}

From January 2010 to August 2011, 14 patients (6 women, median age 64.5 years, range $55-85$ years) with $\mathrm{BAO}$ were treated by endovascular means. The median NIHSS score on admission was 21 (range 5-36). In our sample, $57.1 \%$ of patients had hypertension, $14.3 \%$ had diabetes, $14.3 \%$ were current cigarette smokers, $57.1 \%$ had hypercholesterolemia, $21.4 \%$ had atrial fibrillation, 
$21.4 \%$ had coronary artery disease, and $21.4 \%$ had a history of previous stroke or TIA.

The occlusion site was the proximal basilar artery in $2(14.3 \%)$ patients, the mid-basilar artery in $1(7.1 \%)$ patient, the distal basilar artery in $10(71.4 \%)$ patients, and the proximal to distal basilar artery segments in $1(7.1 \%)$ patient. Stroke etiology, according to TOAST criteria, was large vessel disease in 5 (35.7\%), cardioembolic in $6(42.9 \%)$, and undetermined in $3(21.3 \%)$ patients. Five patients $(35.7 \%)$ received intravenous rtPA as bridging therapy before endovascular treatment. Seven patients (50\%) received intra-arterial thrombolysis (median dose 550,000 IU, range 100,000-1,000,000 IU). Thrombus aspiration prior to using a stent retriever was performed in 4 patients (28.6\%). Mechanical thrombectomy without adjunctive thrombolytic therapy was performed in 4 patients (28.6\%). Delivery of the Solitaire FR was technically feasible in all patients. All patients were treated with the Solitaire FR $4 \times 20 \mathrm{~mm}$, with an average number of passes of 1.3 (range 1-3), until achieving maximal recanalization (1 retrieval attempt in 12 patients, 3 retrieval attempts in 2 patients). Immediate flow restoration after stent deployment was observed in $78.6 \%(11 / 14)$. Overall, TICI 3 or 2 b recanalization grade was achieved in all patients. After stent retrieval, recanalization grade was TICI 3 in 10 patients (71.4\%). In 4 patients (28.6\%), recanalization grade after stent retrieval was insufficient due to an underlying atherosclerotic stenosis. Additional deployment of a permanent intracranial stent was performed in 3 patients $(21.4 \%$; predilation in 2 patients, postdilation in 1 patient) and PTA alone in 1 patient $(7.1 \%)$. These resulted in a final recanalization grade of TICI 3 in 1 patient and TICI $2 b$ in 3 patients.

The median time from symptom onset to first angiogram was 414 minutes (range 176-1440 minutes) and median procedure time to recanalization was 47 minutes (range 10-252). No thromboembolic occlusion of a previously unaffected artery was found at control angiography after stent retrieval. No device-related adverse events were observed.

There were $3(21.4 \%)$ asymptomatic postprocedure hemorrhages according to the PROACT II criteria, ${ }^{22}$ but no clinically symptomatic intracranial hemorrhage occurred.

Median NIHSS score after 24 hours was 8 (range 1-36). Of the total sample, $35.7 \%$ of patients $(5 / 14)$ improved by $>9$ points on the NIHSS scale after 24 hours. Mortality was 35.7\% (5/14). Four of the 9 surviving patients $(28.6 \%)$ had favorable clinical outcomes after 3 months (mRS $0-2)$ and $5(35.7 \%)$ had poor outcomes (mRS 3-5).

\section{DISCUSSION}

The introduction of retrievable stents to the armamentarium of endovascular ischemic stroke treatment increased recanalization rates up to $80 \%-94 \% .^{10-12,15-17}$ Recent preliminary clinical studies examined the treatment effect of retrievable stents in large vessel occlusions, mainly in the anterior cerebral circulation (ICA and MCA occlusions), including only a few or a single acute BAO. Therefore, only very limited data and detailed analyses of the treatment effect of stent retrievers in acute BAO are available in the literature.

Roth et $\mathrm{al}^{12}$ included 8 acute BAOs in their series of 20 intracranial vessel occlusions treated with the Solitaire FR. Recanaliza- tion was successful in all BAOs, defined as a TICI score $>2$, with a mean number of device passes of 1.75. Symptomatic intracranial hemorrhage occurred in 1 patient. Good functional outcome (mRS $0-2$ ) was achieved in 50\% of patients (4/8) and mortality was $37.5 \%$ (3/8). Miteff et $\mathrm{al}^{17}$ presented 10 acute BAOs in their series, totaling 26 patients treated using the Solitaire FR. Presumed etiology of BAO was cardioembolic in 4, dissection in 3, atherosclerosis in 1, iatrogenic in 1 , and unknown in 1 patient. TIMI 2 or 3 recanalization grade was achieved in all patients (TIMI 3 in 33.3\%, 3/10 patients), with more than 3 device passes needed in $50 \%$ of cases. Good functional outcome ( $\mathrm{mRS} 0-2$ ) was observed in $20 \%$ of patients (2/10), and mortality was 33.3\% (3/ 10). The largest number of acute BAOs treated thus far with the Solitaire FR was reported by Costalat et $\mathrm{al},{ }^{15}$ who included 16 acute BAOs in their series of 50 patients with large vessel occlusion stroke. Successful recanalization (defined as TICI 3) was achieved in $81 \%(13 / 16)$, with a mean of 2.1 passes. One patient experienced a symptomatic intracranial hemorrhage, and symptomatic embolic events occurred in 2 patients. An mRS of $0-2$ at 3 months was observed in $44 \%$ (7/16), with a mortality rate of $25 \%(4 / 16)$. The recanalization results of the present study are in line with these previous data, confirming the capability of achieving very high recanalization rates in the setting of a multimodal approach.

However, despite the high recanalization success, favorable clinical outcome was limited. This finding might be attributable to the small sample size of the study. On the other hand, especially in basilar artery occlusions, other factors have been noted as predictors for outcome, such as high initial NIHSS score at presentation, younger age, time span from onset to recanalization, and thrombus extent and location, ${ }^{3,5,6,23-27}$ which may influence clinical outcome in addition to recanalization success.

The main causes of acute BAOs are atherothrombotic occlusions, due to local thrombosis on the ground of a high-grade stenosis, and embolic occlusions, due to cardiac or arterio-arterial thromboembolism. Atherothrombotic lesions are found in around $26 \%-36 \%$ of patients with acute $\mathrm{BAO}$ and are more often localized at the vertebrobasilar junction and up to the midbasilar segment. Embolic occlusions account for around 30\%-35\% of acute BAOs. These occur more often in the distal segment of the basilar artery and are reported to recanalize easier. $.^{3,8,23,27-29} \mathrm{Re}-$ markably, all 4 patients in this study, where insufficient recanalization after stent retrieval was achieved, had an underlying highgrade atherosclerotic basilar artery stenosis. This observation might be attributable to several findings. First, thrombi occurring on the surface of ulcerated atherosclerotic plaques are usually more adhesive than embolic thrombi and therefore more difficult to extract. Second, only partial recanalization after stent retrieval on the grounds of a high-grade atherothrombotic lesion has a higher risk of reocclusion than a completely recanalized vessel. Reocclusion rates after intra-arterial thrombolysis have been reported to be $10 \%-30 \% .^{30,31}$ Furthermore, the Solitaire FR does not provide enough radial outward force during its temporary deployment to overcome a high-grade atherosclerotic stenosis. However, the radial force was sufficient to achieve an immediate flow restoration effect by compressing the thrombus against the vessel wall in most patients (11/14). In several studies, PTA and permanent intracranial stent placement were performed in addi- 
tion to thrombolysis and thrombectomy as an on-demand or rescue procedure, with higher recanalization rates than intravenous or intra-arterial thrombolysis alone, ranging from $70 \%-$ 94\%. ${ }^{23,25,26,32}$ Therefore, PTA and/or permanent intracranial stent implantation at the site of the stenosis is still a treatment option when partial recanalization is achieved with a stent retriever.

In cases of thromboembolic BAO, thrombus load can be comparatively large and therefore resistant to thrombolytic therapy. Different thrombolytic agents at higher dosages have to be administered to dissolve the clot, possibly increasing the risk of hemorrhage. ${ }^{33}$ The use of stent retrievers has the advantage of being able to immediately remove larger clot volumes in case of a cardioembolic occlusion.

Currently, proximal temporary balloon occlusion using a balloon-guide catheter in the ICA is recommended in anterior circulation strokes to avoid embolization or shearing off of thrombus fragments during stent retrieval. However, these guidelines are inconsistently followed in published series, and there are limitations to this approach in the posterior circulation, where a bilateral proximal vertebral artery occlusion with 2 balloon-guide catheters via a bifemoral approach would be necessary to achieve flow control. ${ }^{34,35}$ This renders the procedure more time consuming and complicated. Instead, we attempted to use the largest possible guide catheter allowing safe catheterization of the vertebral artery to achieve flow reduction and to enable effective aspiration during retrieval. With this technique, no thromboembolic events in previously unaffected arteries occurred in this series.

In the present study, no device-related complications occurred and no signs of vessel dissection or perforation were observed on control angiographies. Furthermore, no symptomatic intracranial hemorrhage was encountered after treatment. Only 3 patients with asymptomatic hemorrhages, according to the PROACT II criteria, ${ }^{22}$ were observed on routine posttreatment imaging. This low hemorrhage rate compares favorably with the rates of symptomatic intracranial hemorrhage reported in other stentretriever studies $(0 \% \text { to } 17 \%)^{10,11,15,16}$ and with acute BAO treatment ( $0 \%$ to $19 \%) .8,9,23,24,26,36,37$

This study has inherent limitations due to the retrospective design and the small number of patients. Furthermore, the application of a multimodal approach might confound the treatment effect of mechanical thrombectomy. Therfore, definitive conclusions about the efficacy of stent retrievers in the treatement of acute $\mathrm{BAO}$ cannot be derived from this small study population and further clinical studies are needed.

\section{CONCLUSIONS}

Our preliminary study suggests that a multimodal endovascular approach using retrievable stents in $\mathrm{BAO}$ has high recanalization rates with very low complication rates. Underlying atherothrombotic stenotic lesions of the basilar artery may still necessitate additional permanent stent placement to achieve complete recanalization.

Disclosures: James V. Byrne-UNRELATED: Consultancy: Codman Neurovascular, Comments: Consultancy with no direct relevance to this submission; Grants/Grants Pending: Siemens AG; Other: Teaching collaboration with Stryker Inc, Comments: Unrelated to the subject of this submission. Heinrich P. Mattle-RELATED: Com- ments: Payment for lectures at teaching courses for ev3. UNRELATED: Consultancy: Biogen, Merck, Merck Serono, Novartis, TEVA; Grants/Grants Pending: Scientific grants from the Swiss National Science Foundation and Swiss Heart Foundation; Payment for Lectures (including service on speakers bureaus): Bayer, Biogen, Merck, Merck Serono, Novartis, Pfizer; Royalties: from Thiem for textbooks. Gerhard Schroth-RELATED: Support for Travel to Meetings for the Study or Other Purposes: Star Study steering commission; ${ }^{*}$ UNRELATED: Grants/Grants Pending: The University Institute of Neuroradiology is supported by scientific grants from the Swiss National Foundation (SNF), private foundations (Schebart, Swiss Heart Foundation), and via scientific cooperation contracts from Siemens, Bayer Schering, Bracco, ev3, Invatec. All grants are administrated and controlled by the university hospital; * Payment for Lectures (including service on speakers bureaus): Payment for lectures are part of scientific contracts with the companies mentioned in the previous item and are exclusively paid into the scientific fund of the University Institute of Neuroradiology; Travel/Accommodations/Meeting Expenses Unrelated to Activities Listed: Invited lectures from other European universities, ${ }^{*} \mathrm{Com}$ ments: European Stroke Organization (invited lectures). Jan Gralla-RELATED: Consulting Fee or Honorarium: Covidien, ev3, Comment: Global PI for the STAR study (Solitaire FR in acute Stroke), consultancy for Covidien/ev3; UNRELATED: Consultancy: Arcandis, MicroVention, Comments: Investigator of a core lab for a study by Arcandis. Clinical adviser for MicroVention (LVIS stent); Grants/Grants Pending: Swiss National Fonts. * ( Money paid to institution.)

\section{REFERENCES}

1. Archer CR, Horenstein S. Basilar artery occlusion: clinical and radiological correlation. Stroke 1977;8:383-90

2. Hacke W, Zeumer H, Ferbert A, et al. Intra-arterial thrombolytic therapy improves outcome in patients with acute vertebrobasilar occlusive disease. Stroke 1988;19:1216-22

3. Brandt T, von Kummer R, Muller-Kuppers M, et al. Thrombolytic therapy of acute basilar artery occlusion. Variables affecting recanalization and outcome. Stroke 1996;27:875-81

4. Baird TA, Muir KW, Bone I. Basilar artery occlusion. Neurocrit Care 2004;1:319-29

5. Arnold M, Nedeltchev K, Schroth G, et al. Clinical and radiological predictors of recanalisation and outcome of 40 patients with acute basilar artery occlusion treated with intra-arterial thrombolysis. J Neurol Neurosurg Psychiatry 2004;75:857-62

6. Schulte-Altedorneburg G, Hamann GF, Mull M, et al. Outcome of acute vertebrobasilar occlusions treated with intra-arterial fibrinolysis in 180 patients. AJNR Am J Neuroradiol 2006;27:2042-47

7. Davis SM, Donnan GA. Basilar artery thrombosis: recanalization is the key. Stroke 2006;37:2440

8. Schonewille WJ, Wijman CA, Michel P, et al. Treatment and outcomes of acute basilar artery occlusion in the Basilar Artery International Cooperation Study (BASICS): a prospective registry study. Lancet Neurol 2009;8:724-30

9. Lindsberg PJ, Mattle HP. Therapy of basilar artery occlusion: a systematic analysis comparing intra-arterial and intravenous thrombolysis. Stroke 2006;37:922-28

10. Brekenfeld C, Schroth G, Mordasini P, et al. Impact of retrievable stents on acute ischemic stroke treatment. AJNR Am J Neuroradiol 2011;32:1269-73

11. Castaño C, Dorado L, Guerrero C, et al. Mechanical thrombectomy with the Solitaire $\mathrm{AB}$ device in large artery occlusions of the anterior circulation: a pilot study. Stroke 2010;41:1836-40

12. Roth C, Papanagiotou P, Behnke S, et al. Stent-assisted mechanical recanalization for treatment of acute intracerebral artery occlusions. Stroke 2010;41:2559-67

13. Park H, Hwang GJ, Jin SC, et al. A retrieval thrombectomy technique with the Solitaire stent in a large cerebral artery occlusion. Acta Neurochir 2011;153:1625-31

14. Cohen JE, Leker RR, Moscovici S, et al. Stent-based mechanical thrombectomy in acute basilar artery occlusion. J Clin Neurosci 2011;18:1718-20

15. Costalat V, Machi P, Lobotesis K, et al. Rescue, combined, and standalone thrombectomy in the management of large vessel occlusion stroke using the Solitaire device: a prospective 50-patient singlecenter study: timing, safety, and efficacy. Stroke 2011;42:1929-35

16. Stampfl S, Hartmann M, Ringleb PA, et al. Stent placement for flow 
restoration in acute ischemic stroke: a single-center experience with the Solitaire stent system. AJNR Am J Neuroradiol 2011;32: $1245-48$

17. Miteff F, Faulder KC, Goh AC, et al. Mechanical thrombectomy with a self-expanding retrievable intracranial stent (Solitaire $\mathrm{AB}$ ): experience in 26 patients with acute cerebral artery occlusion. AJNR Am J Neuroradiol 2011;32:1078-81

18. Mordasini P, Frabetti N, Gralla J, et al. In vivo evaluation of the first dedicated combined flow-restoration and mechanical thrombectomy device in a swine model of acute vessel occlusion. AJNR Am J Neuroradiol 2011;32:294-300

19. Higashida RT, Furlan AJ, Roberts $\mathrm{H}$, et al. Trial design and reporting standards for intra-arterial cerebral thrombolysis for acute ischemic stroke. Stroke 2003;34:e109-137

20. van Swieten JC, Koudstaal PJ, Visser MC, et al. Interobserver agreement for the assessment of handicap in stroke patients. Stroke 1988;19:604-07

21. Adams HP Jr, Bendixen BH, Kappelle LJ, et al. Classification of subtype of acute ischemic stroke. Definitions for use in a multicenter clinical trial. TOAST. Trial of Org 10172 in Acute Stroke Treatment. Stroke 1993;24:35-41

22. Kase CS, Furlan AJ, Wechsler LR, et al. Cerebral hemorrhage after intra-arterial thrombolysis for ischemic stroke: the PROACT II trial. Neurology 2001;57:1603-10

23. Jung S, Mono ML, Fischer $U$, et al. Three-month and long-term outcomes and their predictors in acute basilar artery occlusion treated with intra-arterial thrombolysis. Stroke 2011;42:1946-51

24. Renard D, Landragin N, Robinson A, et al. MRI-based score for acute basilar artery thrombosis. Cerebrovasc Dis 2008;25:511-16

25. Kashiwagi J, Kiyosue H, Hori Y, et al. Endovascular recanalization of acute intracranial vertebrobasilar artery occlusion using local fibrinolysis and additional balloon angioplasty. Neuroradiology 2010;52:361-70

26. Chandra RV, Law CP, Yan B, et al. Glasgow coma scale does not predict outcome post-intra-arterial treatment for basilar artery thrombosis. AJNR Am J Neuroradiol 2011;32:576-80

27. Eckert B, Kucinski T, Pfeiffer G, et al. Endovascular therapy of acute vertebrobasilar occlusion: early treatment onset as the most important factor. Cerebrovasc Dis 2002;14:42-50

28. Kubik CS, Adams RD. Occlusion of the basilar artery; a clinical and pathological study. Brain 1946;69:73-121

29. Castaigne P, Lhermitte F, Gautier JC, et al. Arterial occlusions in the vertebro-basilar system. A study of 44 patients with post-mortem data. Brain 1973;96:133-54

30. Becker KJ, Monsein LH, Ulatowski J, et al. Intraarterial thrombolysis in vertebrobasilar occlusion. AJNR Am J Neuroradiol 1996;17: 255-62

31. Zeumer H, Freitag HJ, Zanella F, et al. Local intra-arterial fibrinolytic therapy in patients with stroke: urokinase versus recombinant tissue plasminogen activator (r-TPA). Neuroradiology 1993;35: 159-62

32. Yu YY, Niu L, Gao L, et al. Intraarterial thrombolysis and stent placement for acute basilar artery occlusion. J Vasc Interv Radio 2010;21:1359-63

33. Eckert B, Koch C, Thomalla G, et al. Aggressive therapy with intravenous abciximab and intra-arterial rtPA and additional PTA/ stenting improves clinical outcome in acute vertebrobasilar occlusion: combined local fibrinolysis and intravenous abciximab in acute vertebrobasilar stroke treatment (FAST): results of a multicenter study. Stroke 2005;36:1160-65

34. Mayer TE, Hamann GF, Brueckmann HJ. Treatment of basilar artery embolism with a mechanical extraction device: necessity of flow reversal. Stroke 2002;33:2232-35

35. Clarençon F, Blanc R, Gallas S, et al. Thrombectomy for acute basilar artery occlusion by using double Merci retriever devices and bilateral temporary vertebral artery flow reversal. Technical note. J Neurosurg 2009;111:53-56

36. Pfefferkorn T, Holtmannspotter M, Schmidt C, et al. Drip, ship, and retrieve: cooperative recanalization therapy in acute basilar artery occlusion. Stroke 2010;41:722-26

37. Lutsep HL, Rymer MM, Nesbit GM. Vertebrobasilar revascularization rates and outcomes in the MERCI and multi-MERCI trials. $J$ Stroke Cerebrovasc Dis 2008;17:55-57 